\title{
HAUSDORFF MEASURES AND SETS OF UNIQUENESS FOR TRIGONOMETRIC SERIES
}

\author{
R. DOUGHERTY AND A. S. KECHRIS
}

(Communicated by J. Marshall Ash)

\begin{abstract}
We characterize the closed sets $E$ in the unit circle $\mathbf{T}$ which have the property that, for some nondecreasing $h:(0, \infty) \rightarrow(0, \infty)$ with $h(0+)=0$, all the Hausdorff $h$-measure 0 closed sets $F \subseteq E$ are sets of uniqueness (for trigonometric series). In conjunction with Körner's result on the existence of Helson sets of multiplicity, this implies the existence of closed sets of multiplicity ( $M$-sets) within which Hausdorff $h$-measure 0 implies uniqueness, for some $h$. This is contrasted with the case of closed sets of strict multiplicity ( $M_{0}$-sets), where results of Ivashev-Musatov and Kaufman establish the opposite.
\end{abstract}

A Hausdorff determining function is any function $h:(0, \infty) \rightarrow(0, \infty)$ which is nondecreasing and satisfies $h(0+)=0$. A subset $E$ of $\mathbf{T}$ (the unit circle, viewed here as $\mathbf{R} / 2 \pi \mathbf{Z}$ ) has Hausdorff h-measure 0 if for every $\varepsilon>0$ there is a sequence $\left\{I_{n}\right\}$ of open intervals (arcs) in $\mathrm{T}$ with $E \subseteq \bigcup_{n} E_{n}$ and $\sum h\left(\left|I_{n}\right|\right)<\varepsilon$. (Here $\left|I_{n}\right|=$ (arc length of $I_{n} / 2 \pi$.) It is a well-known theorem of Ivashev-Musatov [2] that metric thinness in the form of Hausdorff $h$-measure 0 cannot imply uniqueness for trigonometric series. More precisely, for any $h$ as above there is a closed set $E$ of Hausdorff $h$-measure 0 which is of restricted multiplicity (we will give a review of terminology below). Kaufman [3; see also 4, VIII.3.3] extended this result by proving that, for any $h$ as above, any closed set of strict multiplicity has a closed subset of Hausdorff $h$-measure 0 which is still of restricted multiplicity. Later Kaufman asked (in a private conversation) whether the same holds for ordinary multiplicity. The main result of this paper gives a negative answer to Kaufman's question, and provides a characterization of those closed sets which, for some $h$, contain no closed subsets of Hausdorff $h$-measure 0 which are of multiplicity.

We now give a brief review of the notation, terminology, and results we need; this material all appears in Kechris-Louveau [4]. Let $K(\mathbf{T})$ be the collection of closed subsets of $\mathbf{T}$. Denote by $U$ the collection of sets $E \in K(\mathbf{T})$ which are of uniqueness (i.e., every trigonometric series converging to 0 on $\mathbf{T} \backslash E$ is identically 0 ), and let $M=K(\mathbf{T}) \backslash U$ (the collection of closed sets of multiplicity). This is

Received by the editors April 21, 1988 and, in revised form, June 1, 1988.

1980 Mathematics Subject Classification (1985 Revision). Primary 42A63, 43A46.

The second author was partially supported by NSF Grant DMS-8718847. 
the classical definition. It can be reformulated in the theory of distributions as follows: A closed set $E \in K(T)$ is in $M$ if it supports no non-0 pseudomeasure whose $n$th Fourier coefficient tends to 0 as $n \rightarrow \pm \infty$. Let $U_{0} \subseteq K(T)$ be the collection of sets of extended uniqueness (i.e., those which support no probability measure whose $n$th Fourier coefficient tends to 0 as $n \rightarrow \pm \infty$ ) and let $M_{0}=K(\mathbf{T}) \backslash U_{0}$ be the collection of closed sets of restricted multiplicity.

Piatetski-Shapiro gave a characterization of sets of uniqueness in terms of Banach spaces. Let $A$ be the space of continuous complex functions on $\mathbf{T}$ with absolutely convergent Fourier series (which is isomorphic to $l_{1}(\mathbf{Z}) \cong c_{0}(\mathbf{Z})^{*}$ ). For $E \in K(\mathbf{T})$, let $I(E)(J(E))$ be the set of members of $A$ which vanish on $E$ (on a neighborhood of $E$ ). In general, $I(E) \neq J(E)$. Then $E$ is in $U$ iff $J(E)$ is weak ${ }^{*}$-dense in $A$ (originally in [7]; see also [4, V.4.1]). Let $U_{1}$ be the collection of $E \in K(T)$ such that $I(E)$ is weak ${ }^{*}$-dense in $A$; then $U \subseteq U_{1} \subseteq U_{0}$, and Körner showed that $U_{1} \neq U$ (originally in [6]; see also [4, VII.3]).

The sets $U$ and $U_{0}$ are closed under countable unions within $K(\mathbf{T})$ (Bari's Theorem; see [4, I.5.1 and II.5]), but $U_{1}$ is not [4, VI.3.6]; let $U_{1}^{*}$ be the set of $E \in K(\mathbf{T})$ which are countable unions of sets in $U_{1}$. Piatetski-Shapiro showed that every set in $U_{1}$ (and hence every set in $U_{1}^{*}$ ) is a countable union of sets in $U_{1}^{\prime}$, where $E \in K(T)$ is in $U_{1}^{\prime}$ iff $I(E)$ is sequentially weak ${ }^{*}$-dense in $A$ (originally in [7; see also 4, VI.2.3]). One has now the following analogue of Kaufman's result: if $h$ is a Hausdorff determining function and $E \in K(\mathbf{T}) \backslash U_{1}^{*}$, then there is $F \subseteq E$ in $M$ with Hausdorff $h$-measure 0 [4, VIII.4.8]. (This is indeed a corollary of a much more general result: If $E \in K(T)$ is such that for every open $V \subseteq \mathrm{T}$ with $E \cap V \neq \varnothing$ we have $\overline{E \cap V} \notin U_{1}$, then every dense $G_{\delta}$ subset of $E$ contains a closed set $F$ in $M$.) Kaufman asked whether one can replace $E \in K(\mathbf{T}) \backslash U_{1}^{*}$ by $E \in M$; we will answer this question negatively by showing that the above property characterizes $U_{1}^{*}$.

Theorem. A set $E \in K(\mathbf{T})$ is in $U_{1}^{*}$ iff there is a Hausdorff determining function $h$ such that every closed subset of $E$ of Hausdorff h-measure 0 is in $U$.

One direction of this has already been noted; for the other, we must show that for every $E \in U_{1}^{*}$ such an $h$ exists. We will use the idea of a construction from Debs-Saint Raymond [1].

Let $S$ be a countable collection of infinite sequences $\left\{a_{n}\right\}_{n=1}^{\infty}$ of positive reals. We say that $E \subseteq \mathbf{T}$ is $S$-small if, for each $\left\{a_{n}\right\} \in S$, there is a sequence $\left\{I_{n}\right\}$ of open intervals such that $E \subseteq \bigcup_{n} I_{n}$ and $\left|I_{n}\right| \leq a_{n}$. Note that, for every Hausdorff determining function $h$, there is a collection $S$ such that any $S$-small $E \subseteq \mathbf{T}$ has Hausdorff $h$-measure 0; just let the $m$ th sequence in $S$ be $\left\{a_{n}^{(m)}\right\}$ where $0<h\left(a_{n}^{(m)}\right)<2^{-n-m}$. Conversely, we have:

Lemma. Given any $S$ as above, there is an $h$ such that any $E \subseteq \mathbf{T}$ which has Hausdorff h-measure 0 is $S$-small. 
Proof. Let the mth sequence in $S$ be $\left\{a_{n}^{(m)}\right\}$, and choose $h$ so that, for all $n$ and $m, h\left(a_{n}^{(m)}\right)>1 / m n$. Suppose $E$ has Hausdorff $h$-measure 0 . Let $\left\{I_{k}\right\}$ be open intervals with $E \subseteq \bigcup_{k} I_{k}$ and $\sum_{k} h\left(\left|I_{k}\right|\right)<1 / m$. We may assume $\left|I_{1}\right| \geq\left|I_{2}\right| \geq \cdots$. Then $\left|I_{n}\right| \leq a_{n}$, because $\left|I_{n}\right|>a_{n}$ would imply $h\left(\left|I_{n}\right|\right) \geq h\left(a_{n}\right)>1 / m n$ and $h\left(\left|I_{n}\right|\right) \geq h\left(\left|I_{2}\right|\right) \geq \cdots \geq h\left(\left|I_{n}\right|\right)>1 / m n$, so $\sum_{k} h\left(\left|I_{k}\right|\right)>n \cdot(1 / m n)=1 / m$, a contradiction. So $E$ is $S$-small.

So assuming $E \in U_{1}^{*}$, it is enough to find $S$ so that

$$
F \subseteq E, F \text { closed , } F S \text {-small } \Rightarrow F \in U .
$$

Since $U$ is closed under countable unions, it is enough to show $(*)$ assuming $E \in U_{1}^{\prime}$. So suppose $f_{m} \in I(E)$ and $f_{m} \rightarrow^{w^{*}} 1$.

Lemma. Let $f \in A, \varepsilon>0$. There is a sequence $\left\{a_{n}\right\}$ of positive reals such that if $F \subseteq Z(f)=\{x \in \mathbf{T}: f(x)=0\}$ and $F \subseteq \bigcup_{n} I_{n}$, where $I_{n}$ are open intervals with $\left|I_{n}\right| \leq a_{n}$, then for some $g \in J(F),\|f-g\|_{A}<\varepsilon$.

Granting the lemma we can complete the proof as follows: Let $\left\{a_{n}^{(m)}\right\}_{n=1}^{\infty}$ be the sequence given by the lemma for $f=f_{m}, \varepsilon=1 / m$. If the $m$ th sequence in $S$ is $\left\{a_{n}^{(m)}\right\}$, and $F \subseteq E$ is $S$-small, then there is $g_{m} \in J(F)$ with $\left\|f_{m}-g_{m}\right\|_{A} \leq 1 / m$. So $g_{m} \rightarrow^{w^{*}} 1$ and hence $J(F)$ is (actually sequentially) weak ${ }^{*}$-dense in $A$, so $F \in U$.

Proof of the lemma. Let $\tau_{x, \delta}$ be the usual trapezoidal function, defined to be 1 on $[x-\delta, x+\delta], 0$ outside $(x-2 \delta, x+2 \delta)$, and linear on $[x-2 \delta, x-\delta]$ and $[x+\delta, x+2 \delta]$. For each $g \in A$, let

$$
N_{g}(\varepsilon)=\min \left\{N \geq 1: \sum_{|n| \geq N}|\hat{g}(n)|<\varepsilon\right\} .
$$

It is not hard to show that

$$
N_{g_{1} g_{2}}(\varepsilon) \leq N_{g_{1}}\left(\frac{\varepsilon}{2\left\|g_{2}\right\|_{A}}\right)+N_{g_{2}}\left(\frac{\varepsilon}{2\left\|g_{1}\right\|_{A}}\right)
$$

for any $g_{1}, g_{2} \in A$. We will now show that, if $g \in A, \delta \in(0,1)$, and $g(x)=0$, then

$$
\delta<\frac{K \varepsilon^{2}}{\|g\|_{A}^{2} \cdot N_{g}(\varepsilon)^{2}} \Rightarrow\left\|g \tau_{x, \delta}\right\|_{A}<8 \varepsilon
$$

for some fixed constant $K$. What is at issue here is that "points obey spectral synthesis." For the reader not familiar with these ideas, we provide the details. First recall the result of Bernstein that, for any trigonometric polynomial $Q$ of degree at most $N,\left\|Q^{\prime}\right\|_{\infty} \leq 2 N\|Q\|_{\infty}$. (One proves this by showing that $Q^{\prime}$ is the convolution of $-2 N Q$ with $K_{N-1}(y) \sin N y$, where $K_{N}(y)=$ $\sum_{|j| \leq N}(1-|j| /(N+1)) e^{i j y}$ is the Féjer kernel.) Also, $\left\|\tau_{x, \delta}\right\| \leq 3[4$, p. 58] and, 
for any $f \in C(\mathbf{T})$ with $f^{\prime} \in L^{2}(\mathbf{T}),\|f\|_{A} \leq|\hat{f}(0)|+C\left\|f^{\prime}\right\|_{L^{2}}$ for some fixed constant $C$ [4, II.2.1]. Now let

$$
Q(y)=\sum_{n \leq|N|} \hat{g}(n) e^{i n y}+\sum_{n>|N|} \hat{g}(n) e^{i n x} ;
$$

then $Q$ is a trigonometric polynomial of degree $\leq N,\|Q\|_{\infty} \leq\|g\|_{A}$, $\|g-Q\|_{A} \leq 2 \sum_{|n|>N}|\hat{g}(n)|,\left\|g \tau_{x, \delta}\right\|_{A} \leq\left\|Q \tau_{x, \delta}\right\|_{A}+\|g-Q\|_{A}\left\|\tau_{x, \delta}\right\|_{A}$, $\left\|Q \tau_{x, \delta}\right\|_{A} \leq\left\|Q \tau_{x, \delta}\right\|_{1}+C\left\|\left(Q \tau_{x, \delta}\right)^{\prime}\right\|_{2},\left\|Q \tau_{x, \delta}\right\|_{1} \leq(4 \delta / 2 \pi)\|Q\|_{\infty}$, $|Q| \leq 2 \delta\left\|Q^{\prime}\right\|_{\infty}$ on $[x-2 \delta, x+2 \delta]$ (since $\left.Q(x)=0\right),\left(Q \tau_{x, \delta}\right)^{\prime}=Q \tau_{x, \delta}^{\prime}+$ $Q^{\prime} \tau_{x, \delta},\left\|Q \tau_{x, \delta}^{\prime}\right\|_{2} \leq K_{1} \delta^{-1 / 2} \sup _{y \in[x-2 \delta, x+2 \delta]}|Q(y)| \leq 2 K_{1}\left\|Q^{\prime}\right\|_{\infty} \sqrt{\delta}$, and $\left\|Q^{\prime} \tau_{x, \delta}\right\|_{2} \leq K_{2}\left\|Q^{\prime}\right\|_{\infty} \delta$; putting these together gives the desired result.

It follows that one can inductively define $a_{1}, a_{2}, \ldots$, such that, for any $x_{1}, x_{2}, \cdots \in Z(f)$, if we let $f_{0}=f, f_{1}=f_{0} \cdot\left(1-\tau_{x_{1}, a_{1}}\right), f_{2}=$ $f_{1} \cdot\left(1-\tau_{x_{2}, a_{2}}\right)=f_{0} \cdot\left(1-\tau_{x_{1}, a_{1}}\right) \cdot\left(1-\tau_{x_{2}, a_{2}}\right), \ldots, f_{n+1}=f_{n} \cdot\left(1-\tau_{x_{n+1}, a_{n+1}}\right), \ldots$, then $\left\|f_{n}-f_{n+1}\right\|_{A}<\varepsilon / 2^{n+1}$.

If now $F \subseteq Z(f)$ and $F \subseteq \bigcup_{n} I_{n}$, where $I_{n}$ is an open interval with $\left|I_{n}\right| \leq$ $a_{n}$, let $k$ be large enough so that $F \subseteq \bigcup_{n \leq k} I_{n}$. We can also clearly assume $F \cap I_{n} \neq 0$ for $n \leq k$. Choose $x_{n} \in F \cap I_{n}$. Let

$$
J_{n}=\left(x_{n}-a_{n}, x_{n}+a_{n}\right) \text {. }
$$

Then $x_{n} \in Z(f)$ and $F \subseteq \bigcup_{n \leq k} J_{n}$. Let $g=f \cdot\left(1-\tau_{x_{1}, a_{1}}\right)\left(1-\tau_{x_{2}, a_{2}}\right) \cdots$ $\left(1-\tau_{x_{k}, a_{k}}\right)$. Then $g=0$ on $\bigcup_{n \leq k} J_{n}$, so $g \in J(F)$, and $\|f-g\|_{A} \leq$ $\sum_{n}\left\|f_{n}-f_{n+1}\right\|_{A}<\varepsilon$.

\section{REFERENCES}

1. G. Debs and J. Saint Raymond, Ensembles boréliens d'unicité au sens large, Ann. Inst. Fourier (Grenoble) 37 (1987), 217-239.

2. O. S. Ivashev-Musatov, M-sets and Hausdorff measure, Soviet Math. Dokl. 3 (1962), 213-216.

3. R. Kaufman, Kronecker sets and metric properties of $M_{0}$-sets, Proc. Amer. Math. Soc. 36 (1972), 519-524.

4. A. S. Kechris and A. Louveau, Descriptive set theory and the structure of sets of uniqueness, London Math. Soc. Lecture Note Series 128, Cambridge Univ. Press, London and New York, 1987.

5. __ Covering theorems for uniqueness and extended uniqueness sets (to appear).

6. T. Körner, A pseudofunction on a Helson set. I, II, Asterisque 5 (1973), 3-224; 231-239.

7. I. I. Piatetski-Shapiro, On the problem of uniqueness of expansion of a function in a trigonometric series and Supplement to the work "On the problem..." ", Gos. Univ. Uc. Zap., 155 Mat. 5 (1952), 54-72 and 165, Mat. 7(1954), 79-97. (Russian)

Department of Mathematics, California Institute of Technology, Pasadena, CaliFORNIA 91125

Current address (R. Dougherty) : Department of Mathematics, University of CaliforNia, Los ANGeles, California 90024 